\title{
Do Urgent Cardiac Cases Utilise More Resources than Elective? A Single Unit Audit
}

\author{
Munir Tarazi*, Nithiananthan Mayooran, Muhammad Nadeem Anjum, Bejoy Philip, Kishore Doddakula \\ Department of Cardiothoracic Surgery, Cork University Hospital, Cork, Ireland
}

"Corresponding author: Munir Tarazi, Cork University Hospital, Wilton, Co. Cork, Ireland. Tel: +447400266 879; Email: munirtarazi@rcsi.ie

Citation: Tarazi M, Mayooran N, Anjum MN, Philip B, Doddakula K(2017) Do Urgent Cardiac Cases Utilise More Resources than Elective? A Single Unit Audit. J Surg:JSUR-155. DOI: 10.29011/JSUR-155.000055

Received Date: 10 July, 2017; Accepted Date: 07 August 2017; Published Date: 14 August, 2017

\section{Abstract}

Introduction: Literature supports that elective cases perform better by utilising minimal resources compared to urgent cases. We performed a study to evaluate if the traditional wisdom is applicable in today's practice.

Methods: 1203 patients undergoing elective and urgent coronary artery bypass grafting from 2010 to 2013 were included. Data was acquired from the theatre register and Patient Analysis Tracking System. Length of stay was considered as the marker for resource utilization. Length of stay greater than 30 days, emergency and salvage patients were excluded.

Results: There is a significant increase in urgent cases from 2010 to 2013 and a decrease of electives during the same period.

Conclusion: Our study demonstrates that urgent cases can be performed with little impact on resources. It also highlights that in our population urgent cases are increasing compared to electives.

Keywords: Cardiac Surgery; Length of Stay; Resources

\section{Introduction}

The Irish health care system gives universal access to hospital and physician services to all citizens irrespective of social, racial, and economic issues. This approach to health care delivery has many social advantages, but difficult decisions about resource allocation and rationing are being considered to control rising health care expenditure.Medical and social benefits of open-heart surgery to treat valvular and ischaemic heart disease have been clearly demonstrated. Analyses of cost versus benefit, quality of life, and long-term survival have supported the role of surgery in the treatment of acquired heart disease.In Ireland, the cost of cardiac cases is calculated by grouping procedures to DRG (Diagnosis Related Group) with the help of PLC (Patient Level Costing). PLC is a mechanism that measures the individual cost of each patient journey through the hospital. DRG is a collection of similar procedures which are grouped together and an average cost is derived through PLC.

Cardiac cases in our centre are divided into 3 categories: Elective, Urgent, and Emergency. Elective cases are surgeries that are scheduled in advance and performed at convenient time. Urgent cases are surgeries performed after the patient becomes medically stable during their inpatient stay after primary admission. Emergency cases are surgeries performed without delay. Almost all of these admissions arrive through emergency department or transfer from a peripheral hospital.Traditional belief and literature supports that, well planned elective cases performed at a convenient time do better and are more economical compared to urgent cases. In the recent years there is a significant increase in urgent cases. This has provoked us to perform a study to see their impact on patient outcome and resources.

\section{Methods}

1203 consecutive patients undergoing elective and urgent cardiac surgery between 2010 and 2012 were included in our study. Data was acquired from the theatre register and Patient Analysis Tracking System (PATS).

\section{Exclusion Criteria}

Post-operative length of stay greater than 30 days, emergency and salvage cases.Length of stay was considered as the marker for resource utilization. 
Citation: Tarazi M, Mayooran N, Anjum MN, Philip B, Doddakula K(2017) Do Urgent Cardiac Cases Utilise More Resources than Elective? A Single Unit Audit. J Surg:JSUR-155.

\section{Results}

As shown in (Table 1).

\begin{tabular}{|c|c|c|c|}
\hline & $\mathbf{2 0 1 0}$ & $\mathbf{2 0 1 1}$ & $\mathbf{2 0 1 2}$ \\
\hline Elective & 244 & 194 & 161 \\
\hline Urgent & 168 & 204 & 232 \\
\hline
\end{tabular}

Table 1: The number of elective and urgent cardiac cases performed in 2010, 2011, and 2012.

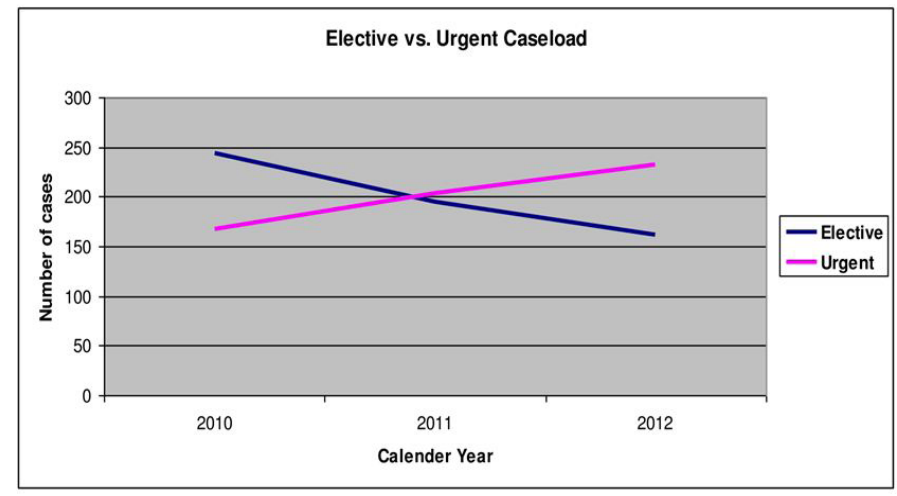

Graph 1: A line graph showing the trend of the number of cardiac cases performed of urgent and elective cardiac cases between 2010 and 2012.

A comparison between the length of stay of urgent and elective cardiac cases is shown in (Graph 2, 3,4).

\section{0}

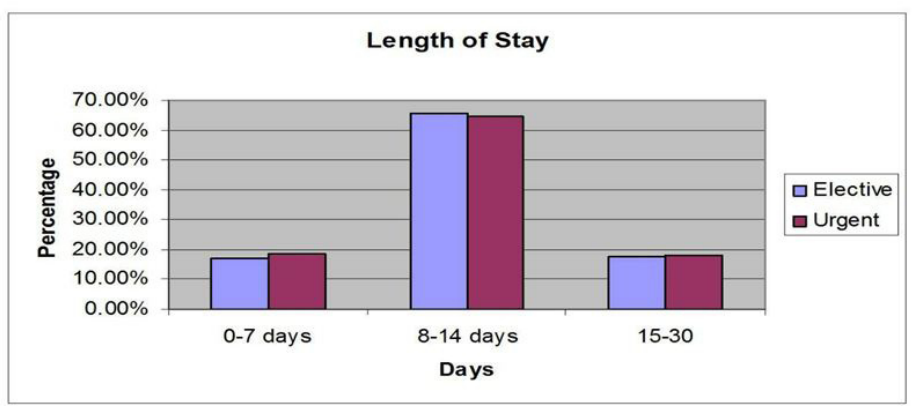

Graph 2: A bar graph showing a comparison of length of stay between urgent and elective cardiac cases in 2010.

In 2010, length of stay was similar between urgent and elective cardiac cases. Around $65 \%$ of cases in both categories were discharged between days 8 and 14 post-operatively.In 2011 and 2012, the trend of similar length of stay between urgent and elective cardiac cases continued. The majority of discharges continued to be between days 8 and 14 post-operatively, though this decreased to around $50 \%$ with a noticeable increase in discharges occurring within the first 7 days post-operatively.

\section{1}

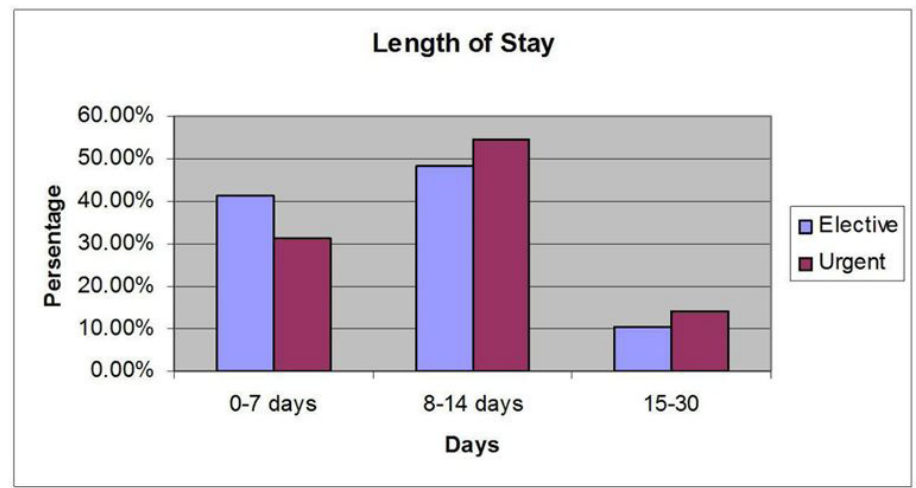

Graph 3: A line graph showing the trend of the number of cardiac cases performed of urgent and elective cardiac cases between 2010 and 2012.

\section{2}

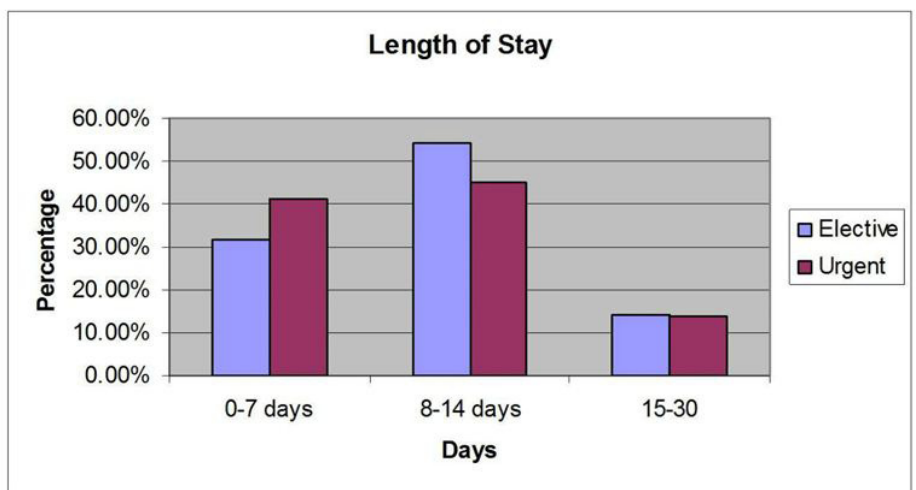

Graph 4: A bar graph showing a comparison of length of stay between urgent and elective cardiac cases in 2012.

\section{Discussion}

Traditional belief and literature supports that elective cardiac cases perform better and are more economical than urgent cardiac cases. Leung et al reported that pre-operative hospital length of stay was a risk factor for post-operative mediastinitis [1]. Urgent cardiac cases have a longer pre-operative length of stay than elective cases. Developing post-operative mediastinitis increases patient length of stay thus increasing cost and resource utilisation. Kerbage et al found that an urgent and emergent cardiac case was an independent predictor of long hospital stay post cardiac surgery [2]. Similarly, Tu et al showed that urgent cases had a statistically significant higher in-hospital mortality rate, longer mean ICU length of stay, and longer mean post-operative length of stay compared to elective cases [3]. Moreover, Carrier et al showed that the length of waiting time for an elective case did not affect 
Citation: Tarazi M, Mayooran N, Anjum MN, Philip B, Doddakula K(2017) Do Urgent Cardiac Cases Utilise More Resources than Elective? A Single Unit Audit. J Surg:JSUR-155.

patient outcomes or length of stay. Furthermore, mortality rate between urgent and elective cases was similar, though; post-operative complications were significantly higher in the urgent group [4].As shown previously, much of the previous literature supports a longer length of stay in urgent cardiac cases compared to elective cardiac cases. Our data showed 3 main outcomes: 1) in recent years, there has been a significant increase in urgent cardiac cases with a decrease in elective cardiac cases. 2) Length of stay between urgent and elective cardiac cases was similar between 2010 and 2012. 3) There has been a noticeable increase in discharges occurring within the first 7 days with a decrease between day 8-14 and $>14$ days.

Nowadays, with improved surgical technique, improved bypass machines, improved anaesthetic and nursing care, and improved physiotherapy involvement and support, electiveand urgent cardiac cases are doing better post-operatively explaining the increase in discharges in the first 7 days.Generally, urgent and elective cardiac cases undergo very similar operations with similar operative time and cardiopulmonary bypass time. This goes to explaining why outcomes in terms of post-operative length of stay and resource utilisation have been similar in both urgent and elective cardiac cases.It is important to note that our data demonstrates that there is no difference in resource utilisation between urgent and elective cardiac cases intra- and post-operatively. Though, overall resource utilisation will be higher in urgent cardiac cases due to their pre-operative hospital stay and use of inpatient cardiology testing such as echocardiography, carotid Doppler ultrasound, and coronary angiography, in some cases during emergency hours.

\section{Conclusion}

Our data demonstrates that urgent cases can be performed with little impact on resources intra- and post-operatively. Overall resource utilization will be higher in the urgent cases due to preoperative hospital stay. Our study also highlights that in our cohort of population, urgent cases are increasing in number as compared to elective cases.

\section{References}

1. Leung Wai Sang S, Chaturvedi R, Alam A, Samoukovic G, de Varenees $B$, et al. (2013) Preoperative hospital length of stay as a modifiable risk factor for mediastinitis after cardiac surgery. Journal of Cardiothoracic Surgery 8.

2. S Kerbage, G Giunta, E Gallucci, H Raffaelli, J Abud, et al. (2010) Predictors of Long Hospital Stay After Cardiac Surgery. European Heart Journal 3: 61 .

3. Tu JV, Jaglal SB, Naylor CD (1995) Multicenter validation of a risk index for mortality, intensive care unit stay, and overall hospital length of stay after cardiac surgery. Circulation 91: 677-684.

4. Carrier M, Pineault R, Tremblay N, Pelletier LC (1993) Outcome of rationing access to open-heart surgery: effect of the wait for elective surgery on patient outcome. Canadian Medical Association Journal 149: $1117-1122$ 
Citation: Tarazi M, Mayooran N, Anjum MN, Philip B, Doddakula K(2017) Do Urgent Cardiac Cases Utilise More Resources than Elective? A Single Unit Audit. J Surg:JSUR-155. 